\title{
EDITORIAL SPECIAL TOPIC Digital is the New Normal: The Role of Digital Media during the COVID-19 Crisis
}

\author{
Oswald D. Kothgassner ${ }^{1} \&$ Thomas Probst ${ }^{2}$ \\ 1 Department of Child and Adolescent Psychiatry, Medical University of Vienna \\ ${ }^{2}$ Department for Psychotherapy and Biopsychosocial Health, Danube University Krems
}

DOI 10.24989/dp.v1i2.1935

We hope you are all well despite the current situation with the COVID-19 pandemic. In 2020, the daily lives of people worldwide have changed. The outbreak of SARS-CoV2 and the necessary measures to contain it (Nussbaumer-Streit et al., 2020) have caused emotional distress and increased the risk of negative mental health outcomes for broad sections of the population (Vindegaard \& Benros, 2020). In Austria, for example, data indicates that mental health problems increased during lockdown as compared to before the pandemic (Pieh, Budimir \& Probst, 2020). Moreover, there were more new onsets of depression than remissions following the lifting of lockdown restrictions (Probst, Budimir \& Pieh, 2020). During the crisis caused by the SARS$\mathrm{CoV} 2$ virus, the process of digitalization has become more prominent. For example, health care providers had to shift from provision of in-person care to remote treatments via digital media in order to maintain mental health care services. The Spanish flu caused a comparable worldwide pandemic 100 years ago, but this is the first time we are faced with such a situation since the rise of globalization. This is why the second issue of Digital Psychology includes a special topic about how COVID-19 changed our digital media behavior. We made the inclusion because the current situation led to a proliferation of COVID-19-related research, and the changes and challenges in digitalization are manifold in the face of this crisis.

The special topic consists of four high-quality and interesting articles addressing a variety of topics related to COVID-19. In this issue, experts Martina Zemp and Helmut Hlavacs discuss technological developments in the fight against COVID-19 as well as the problems of homeschooling and remote work. The Spotlight communication by Humer and Probst (2020) focuses on the transition to remote psychotherapy during the COVID-19 crisis across different countries. Another Spotlight communication by Huscsava et al. (2020) reflects the perspectives of psychiatric patients on remote therapy during the pandemic using a qualitative approach. Further, as the pandemic provokes the dissemination and proliferation of misinformation about COVID-19 in social media, the contribution by Goreis and Kothgassner (2020) provides an overview of current research on conspiracy beliefs in the context of COVID-19, and explores containment of misinformation in social media. Finally, Mon- tag et al. (2020) report on the benefits and potential of digital phenotyping to gain insights into the mental health of the general population during a pandemic, and how it can contribute to decision-making processes of policy makers.

We want to thank all the authors and reviewers for their hard work on this special topic. Moreover, we thank our readers for their interest in this thematic issue.

Oswald D. Kothgassner \& Thomas Probst Topic Editors

\section{References}

Goreis, A. \& Kothgassner, O. D. (2020). Social Media as a vehicle for conspiracy beliefs about COVID-19. Digital Psychology 1(2), 36-39.

Humer, E. \& Probst, T. (2020). Provision of Remote Psychotherapy during the COVID-19 Pandemic. Digital Psychology 1(2), 27-31.

Huscsava, M., Plener, P., \& Kothgassner, O. D. (2020). Teletherapy for adolescent psychiatric outpatients: the soaring flight of so far idle technologies during the COVID-19 pandemic. Digital Psychology 1(2), 32-35.

Montag, C., Dagum, P., \& Elhai, J. D. (2020). On the need for digital phenotyping to obtain insights into mental states in the COVID-19 pandemic. Digital Psychology 1(2), 40-42.

Nussbaumer-Streit B, Mayr V, Dobrescu AI, Chapman A, Persad E, Klerings I, Wagner G, Siebert U, Christof C, Zachariah C \& Gartlehner, G. (2020). Quarantine alone or in combination with other public health measures to control COVID-19: A rapid review. Cochrane Database Systematic Reviews, 2020, CD013574.

Pieh, C., Budimir, S., Probst, T. (2020). The effect of age, gender, income, work, and physical activity on mental health during coronavirus disease (COVID-19) lockdown in Austria. Journal of Psychosomatic Research, 136: 110186.

Probst, T., Budimir, S., Pieh, C. (2020). Depression in and after COVID-19 lockdown in Austria and the role of stress and loneliness in lockdown: A longitudinal study. Journal of Affective Disorders, 277, 962-963.

Vindegaard, N., \& Benros, M. E. (2020). COVID-19 pandemic and mental health consequences: Systematic review of the current evidence. Brain, Behavior, and Immunity, S0889159120309545. 